\title{
Enhancing Security in VLC Systems Through Beamforming
}

\author{
Sunghwan Cho*, Gaojie Chen ${ }^{\#}$, and Justin P. Coon* \\ *Department of Engineering Science, University of Oxford, OX1 3PJ, United Kingdom. \\ \{sunghwan.cho and justin.coon\}@eng.ox.ac.uk \\ \#Department of Engineering, University of Leicester, LE1 7RH, United Kingdom. \\ gaojie.chen@leicester.ac.uk
}

\begin{abstract}
This paper proposes a novel zero-forcing (ZF) beamforming strategy that can simultaneously cope with active and passive eavesdroppers (EDs) in visible light communication systems. A related optimization problem is formulated to maximize the signal-to-noise ratio (SNR) of the legitimate user (UE) while suppressing the SNR of active ED to zero and constraining the average SNR of passive EDs. The proposed beamforming directs the transmission along a particular eigenmode related to the null space of the active ED channel and the intensity of the passive ED point process. An inverse free preconditioned Krylov subspace projection method is used to find the eigenmode. The numerical results show that the proposed $\mathrm{ZF}$ beamforming scheme yields better performance relative to a traditional $\mathrm{ZF}$ beamforming scheme in the sense of increasing the SNR of the UE and reducing the secrecy outage probability.
\end{abstract}

Index Terms-Physical layer security, visible light communication, zero-forcing, active eavesdropper, passive eavesdroppers.

\section{INTRODUCTION}

Recently commercialized 5G technology will support the emergence of new digital services and technology, such as virtual/augmented reality, autonomous driving, Internet of Things, and wireless backhaul. Now that $5 \mathrm{G}$ has passed well into development, it is natural to look toward the future at technologies that will improve data rates and lower latencies even beyond what is promised by 5G. To fulfill this goal, one may find it prudent to consider communication solutions at higher frequencies than those used by $5 \mathrm{G}$, perhaps encompassing a combination of the $1-3 \mathrm{GHz}$ band and the millimeterwave (mmWave) band $(30300 \mathrm{GHz})$, but also straying into the terahertz band $(0.0610 \mathrm{THz})[1]$.

One focus area in beyond-5G research is the utilization of the visible light spectrum for communication in order to exploit this broad patch of largely untouched, unlicensed bandwidth [2]. Visible light communication (VLC) has been rigorously studied among researchers and engineers with a view to implemention and commercialization in future wireless systems. Recently, the standardization of VLC has been conducted by the Visible Light Communication Consortium (VLOC) in Japan and the IEEE in the United States, which have published four VLC standards: Japan Electronics and Information Technology Industries Association (JEITA) CP1221, JEITA CP-1222, JEITA CP1223, and IEEE 802.15.7 [3]. VLC has several notable advantages that can offer significantly higher capacity due to broader modulation bandwidth, high area spectral efficiency, reduced cost of wireless communications due to the unlicensed spectrum, and energy-saving since the VLC transmitters can be simultaneously used for illumination and communications. Lastly, VLC can be useful for achieving secure data transmission thanks to the intrinsic property that visible light cannot penetrate opaque objects [4].

Despite the higher security of VLC, a significant obstacle to wide-scale deployment remains. Specifically, an eavesdropper (ED) can wiretap the transmitted information in large open spaces, such as libraries, offices, and shopping malls. To augment the security of VLC transmissions, various physical layer security (PLS) techniques - such as beamforming, jamming, and light-emitting diode (LED) selection [5]-[10] have been proposed, and their secrecy performances have been analytically verified. Here, PLS denotes a set of methods that utilize the channel randomness to transmit a message with a well-designed coding technique, which allows appropriate redundancy so that only the legitimate user (UE) can reliably decode the message. In contrast, it also introduces enough randomness so that an ED experiences significant uncertainty when attempting to decode the message [11].

However, the primary weakness of existing VLC PLS techniques is that their designs are based upon the assumption that either active or passive EDs are present, but not both simultaneously. The difference between active and passive EDs in VLC systems lies with the availability of the channel state information (CSI) of the EDs at the transmitter sites. In reality, since EDs may try to escape the vigilance of a UE or a network manager, identifying the types, locations, and numbers of the EDs would be impractical. A common assumption is that only active EDs (AEDs) are present in the neighborhood of the transmitter; see, e.g., [5]-[8]. Under this assumption, the design objective is generally to suppress the signal received at the AEDs' sites by steering a transmission beam in the direction of the null space of the AEDs' channels. However, if unknown passive EDs (PEDs) are concurrently present, these PLS schemes may yield opportunities for eavesdropping to the PEDs. In contrast, the previous PLS schemes devised for PEDs have ignored the possible existence of an AED [9], [10], which can be regarded as a more obvious security threat than PEDs. Hence, PLS must be further improved to treat all types of ED simultaneously. 


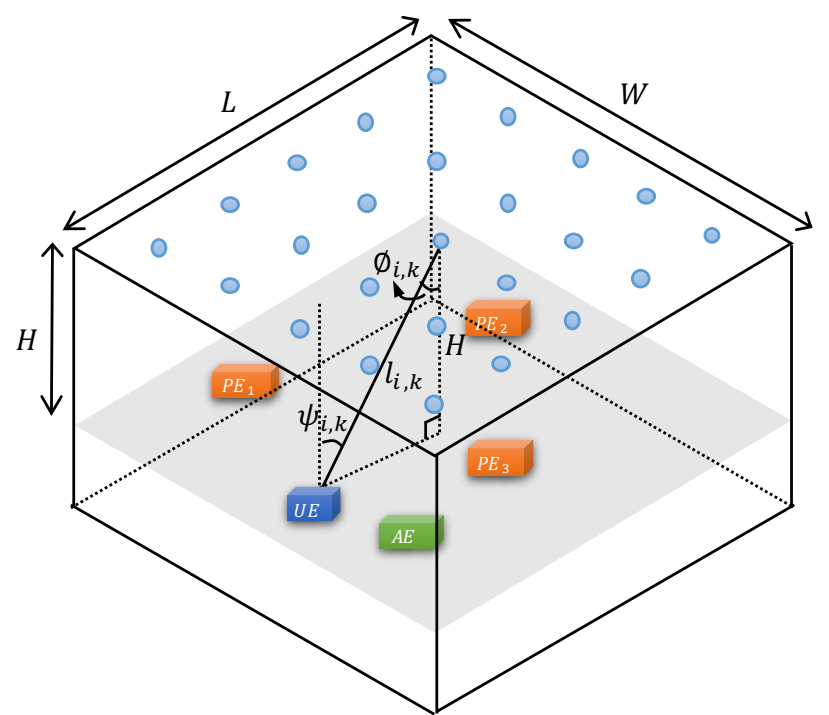

Fig. 1. Rectangular room configuration for VLC systems.

In this paper, we propose a novel zero-forcing (ZF) beamforming scheme that can cope with active and passive EDs simultaneously. We formulate an optimization problem to find a beamforming weight vector that lies in the null space of the AED channel while maximizing the signal-to-noise ratio (SNRs) of the UE and constraining the average SNR of the PEDs. The proposed beamforming scheme directs the transmission along a particular eigenmode related to the null space of the AED channel and the intensity of the PED point process. We adopt an inverse free preconditioned Krylov subspace projection method [12] to estimate the eigenmode iteratively.

The rest of this paper is organized as follows. Section II begins with the system model describing the modulation and beamforming schemes in VLC. In Section III, a novel ZF beamforming design is proposed based on the SNR of the receivers. Section IV gives numerical results that verify our analysis. Section V concludes the paper.

\section{System Model}

As shown in Fig. 1, we consider the downlink of a multipleinput single-output (MISO) VLC system, which consists of $N$ LED transmitters, one UE, one AED, and multiple PEDs. The LED transmitters are attached to the ceiling of the room, and each LED transmitter, i.e., an LED fixture consisting of multiple individual LEDs, is assumed to be capable of communicating independently of other transmitters. Also, all the receivers are assumed to be located in the (gray) work plane of the room. Here, $W, L$, and $H$ denote the width, the length, and the height of the ceiling relative to the work plane. We also assume that the LED transmitters know the locations of the UE and the AED, while the locations of the PEDs are not available. Instead, the statistical information of the PED locations is assumed to be available, that is, the PEDs are assumed to be randomly and uniformly distributed according to homogeneous Poisson Point Process (PPP) $\Phi_{P E}$ with density $\lambda_{P E}$. Note that the homogeneous PPP distribution of PEDs implies that their locations are completely unknown, which is the worst case from the secrecy viewpoint [13]. The $\mathrm{UE}$, the AED, and the PEDs are denoted by $U, A E$, and $P E_{e}$ for $e \in \Phi_{E}$, respectively. Also, we assume that all of the EDs act independently of one another, i.e., there is no collusion.

A direct-current (DC) biased pulse-amplitude modulation VLC scheme is considered [5]. The data signal $s_{i}(t) \in \mathbb{R}$ for the $i$ th transmitter in time slot $t$ is superimposed on a fixed bias current $I_{D C} \in \mathbb{R}_{+}$. The current $I_{D C}$ is used for the purpose of illumination. Thus, the $i$ th transmitter's modulated signal $x_{i}(t)$ corresponding to $s_{i}(t)$ is a zero-mean current signal that can be expressed as $x_{i}(t)=\alpha I_{D C} s_{i}(t)$, where $\alpha \in[0,1]$ is the modulation index. To maintain linear current-to-light conversion, the amplitude of $x_{i}(t)$ is constrained such that $\left|x_{i}(t)\right| \leq \alpha I_{D C}$. Also, $\mathbb{E}\left[x_{i}(t)\right]=0$ is assumed; thus the data signal does not affect illumination.

According to [14], the channel gain $h_{i, k} \in \mathbb{R}_{+}$from the $i$ th transmitter to the receiver $k \in\left\{U, A E, P E_{e}\right\}$ corresponding to an LED with a generalized Lambertian emission pattern in a VLC system is given by $h_{i, k}=\eta G_{i, k} R_{k} T_{k}$, where $\eta$ (W/A) is the current-to-light conversion efficiency, and $R_{k}$ $(\mathrm{mA} / \mathrm{mW})$, is the photodetector's responsivity, and $T_{k}$ (V/A) is the transimpedance amplifier gain. Also, $G_{i, k}$ denotes the Line-of-Sight (LoS) path gain, which is given by

$$
\begin{aligned}
& G_{i, k}= \\
& \left\{\begin{array}{cll}
\frac{(m+1) A_{\mathrm{PD}, k}}{2 \pi l_{i, k}^{2}} \frac{\kappa_{k}^{2} \cos ^{m}\left(\phi_{i, k}\right)}{\sin ^{2}\left(\Psi_{c}\right)} \cos \left(\psi_{i, k}\right) & \text { for } & \left|\psi_{i, k}\right| \leq \Psi_{c} \\
0 & \text { for } & \left|\psi_{i, k}\right|>\Psi_{c}
\end{array}\right.
\end{aligned}
$$

where $m=-\ln (2) / \ln \left(\cos \left(\phi_{1 / 2}\right)\right)$ is the order of Lambertian emission with half illuminance at $\phi_{1 / 2}$, and $A_{\mathrm{PD}, k}\left(\mathrm{~m}^{2}\right)$ is the physical area of the photodiode (PD) at the receiver. As shown in Fig. $1, l_{i, k}(\mathrm{~m})$ denotes the distance between the $i$ th transmitter and the receiver $k . \phi_{i, k}$ is the angle of irradiance, and $\psi_{i, k}$ is the angle of incidence. $\kappa_{k}$ is the refractive index of the optical concentrator, and $\Psi_{c}$ denotes the received field of view of the PD. By assuming that a receiver's PD faces up normal to the work plane, i.e., assuming $\phi_{i, k}=\psi_{i, k}$, we can rewrite the channel gain as $h_{i, k}=K l_{i, k}^{-(m+3)}$, where $K=\left(\eta(m+1) A_{\mathrm{PD}, k} H^{m+1} \kappa_{k}^{2} R_{k} T_{k}\right) /\left(2 \pi \sin ^{2}\left(\Psi_{c}\right)\right)$.

Similarly to [5], [9], a beamforming vector $\mathbf{w}=$ $\left[\omega_{1}, \omega_{2}, \ldots, \omega_{N}\right]^{T}$ can be defined, where $\omega_{i}$ for $i \in\{1,2, \ldots, N\}$ is a weight for the $i$ th transmitter $\left(\left|\omega_{i}\right| \leq 1\right)$. The superscript $T$ denotes the transpose operation. Each LED transmitter multiplies the modulated data signal $x_{i}(t)$ by $\omega_{i}$ before emitting the signal. Therefore, the received signal at receiver $k$ in the considered MISO VLC system can be expressed as

$$
y_{k}(t)=\alpha I_{D C} \mathbf{h}_{k}^{T} \mathbf{w} s(t)+n_{k}(t)
$$

where $\mathbf{h}_{k}=\left[h_{1, k}, h_{2, k}, \ldots, h_{N, k}\right]^{T} \in \mathbb{R}^{N}$ denotes the channel gain vector. In the considered MISO VLC system, the data 
signal $s_{i}(t)$ for the $i$ th transmitter is assumed to be identical, i.e., $s_{i}(t)=s(t)$ for all transmitters (see [5], [9]). Also, $n_{k}$ signifies a zero-mean additive white Gaussian noise (AWGN) random variable at receiver $k$ with variance $\sigma^{2}$. For notational convenience, the time index $t$ is ignored for the remainder of the paper.

For Gaussian VLC MISO channels with an amplitude constraint, we define the peak SNR, rather than the average, by assuming $s=1$ since the channel capacity bounds of the VLC systems are described as a function of the peak SNR [15]. The peak SNR at the receiver $k$ with beamforming can be written as

$$
\gamma_{k}=\frac{\alpha^{2} I_{D C}^{2} \mathbf{w}^{T} \mathbf{h}_{k} \mathbf{h}_{k}^{T} \mathbf{w}}{\sigma^{2}}=\varphi \mathbf{w}^{T} \mathbf{H}_{k} \mathbf{w}
$$

where $\mathbf{H}_{k}=\mathbf{h}_{k} \mathbf{h}_{k}^{T}$ and $\varphi=\alpha^{2} I_{D C}^{2} / \sigma^{2}$.

Also, as in [16], we define the secrecy outage probability (SOP) as the probability that the SNR of the UE is lower than the maximum SNR observed by EDs, which is described as

$$
P_{S O} \stackrel{(a)}{=} \mathbb{P}\left(\gamma_{U}<\max _{e \in \Phi_{E}} \gamma_{P E_{e}}\right) \stackrel{(b)}{=} 1-\exp \left(-\iint_{\mathcal{A}} \lambda_{E} \mathrm{~d} \mathbf{x}\right) .
$$

For (a), it is assumed that secrecy outage occurs only when the maximum SNR observed by a PED is greater than the SNR of the UE, which is a reasonable assumption since the SNR of the AED is suppressed to zero by using the proposed beamforming design. For (b), we apply the void probability of a PPP [17], where $\mathcal{A}=\left\{\mathbf{x} \mid \gamma(\mathbf{x}, \mathbf{w})>\gamma_{U}\right\}$ denotes the area in which the SNR is greater than that of the UE. Here, $\gamma(\mathbf{x}, \mathbf{w})$ denotes the SNR measured at the point $\mathbf{x} \in \mathbb{R}^{2}$ in the work plane of the room (the center of the room is the origin of our coordinate system), which can be expressed as

$$
\gamma(\mathbf{x}, \mathbf{w})=\varphi \mathbf{w}^{T} \mathbf{h}(\mathbf{x}) \mathbf{h}(\mathbf{x})^{T} \mathbf{w}
$$

where $\mathbf{h}(\mathbf{x})$ denotes the channel gain vector at the point $\mathbf{x}$.

\section{ZERO-ForCING BEAMFORMING AGAINST ACTIVE AND PASSIVE EAVESDROPPERS}

In this section, we propose beamformer designs based on the formulation of several optimization problems that aim to improve secrecy performance when an AED and PEDs are simultaneously present.

\section{A. Maximizing User SNR}

In the traditional ZF beamforming for VLC systems [6], the beamforming vector can be obtained by projecting the UE's channel onto the null space of the AED, which can be expressed as

$$
\mathbf{w}=g \Psi_{A E} \Psi_{A E}^{T} \mathbf{h}_{U} .
$$

Here, $\Psi_{A E} \in \mathbb{R}^{N \times(N-1)}$ is a matrix whose $N-1$ columns constitute a basis for the null space of the AED's channel $\mathbf{h}_{A E}$ and $g$ is a constant such that $|\mathbf{w}| \leq \mathbb{1}$. Note that the PEDs are ignored in the traditional $\mathrm{ZF}$ beamforming design. By laying the beamforming vector in the null space of the AED channel, the LED transmitters can make the SNR of the
AED zero. Based on this idea, in this subsection, we find a new ZF beamformer that lies in the null space of the AEDs channel in order to suppress the SNRs of the AED to zero while simultaneously maximizing the SNRs of the UE and constraining the average SNR of the PEDs.

First, since the LEDs do not retain the knowledge of PED locations, it is a natural approach to consider the average SNR of a PED, which can be expressed by

$$
\begin{aligned}
\bar{\gamma}_{P E} & =\mathbb{E}\left[\varphi \mathbf{w}^{T} \mathbf{h}_{P E_{e}} \mathbf{h}_{P E_{e}}^{T} \mathbf{w}\right] \\
& =\varphi \mathbf{w}^{T} \mathbb{E}\left[\mathbf{h}_{P E_{e}} \mathbf{h}_{P E_{e}}^{T}\right] \mathbf{w}=\varphi \mathbf{w}^{T} \overline{\mathbf{H}}_{P E} \mathbf{w}
\end{aligned}
$$

where $\overline{\mathbf{H}}_{P E}=\mathbb{E}\left[\mathbf{h}_{P E_{e}} \mathbf{h}_{P E_{e}}^{T}\right]$ and its element in the $i$ th row and $j$ th column is given by

$$
\bar{H}_{P E_{i, j}}=\frac{1}{N_{P E}} \int_{\frac{-L}{2}}^{\frac{L}{2}} \int_{\frac{-W}{2}}^{\frac{W}{2}} \frac{\lambda_{P E} K^{2}}{l_{i}^{m+3}(\mathbf{x}) l_{j}^{m+3}(\mathbf{x})} \mathrm{d} \mathbf{x}
$$

where $l_{i}(\mathbf{x})$ denotes the distance between the $i$ th transmitter and the point $\mathbf{x}$. Also, $N_{P E}$ denotes the average number of PEDs in the room, which is given by

$$
N_{P E}=\int_{\frac{-L}{2}}^{\frac{L}{2}} \int_{\frac{-W}{2}}^{\frac{W}{2}} \lambda_{P E} \mathrm{~d} \mathbf{x}
$$

Hence, the optimization can be formulated as

$$
\begin{aligned}
& \arg \max _{\mathbf{a}} \varphi \mathbf{w}^{T} \mathbf{H}_{U} \mathbf{w} \\
& \text { s.t. }\left\{\begin{array}{l}
\mathbf{w}=\Psi_{A E} \Psi_{A E}^{T} \mathbf{a} \\
\varphi \mathbf{w}^{T} \overline{\mathbf{H}}_{P E} \mathbf{w} \leq \bar{\rho}_{P E} \\
|\mathbf{w}| \leq \mathbb{1} .
\end{array}\right.
\end{aligned}
$$

where $\mathbf{a} \in \mathcal{R}^{N}$ is an arbitrary real vector, and $\bar{\rho}_{P E}$ denotes the target constraint on the average SNR of a PED. Note that the objective in (9a) is a function of the optimization variable a due to the relation specified in the first constraint, which ensures that the beamforming vector $\mathbf{w}$ derived by projecting a onto the null space of the AED's channel makes the SNR of the AED zero. Also, for PEDs distributed by homogeneous PPP, restricting the average SNR of a single PED in the second constraint is directly related to constraining the sum of the average SNRs of multiple PEDs as

$$
\begin{aligned}
\mathbb{E}_{\Phi_{P E}}\left[\sum_{l \in \Phi_{P E}} \gamma_{P E_{l}}\right] \stackrel{(a)}{=} \int_{\frac{-L}{2}}^{\frac{L}{2}} \int_{\frac{-W}{2}}^{\frac{W}{2}} \lambda_{P E} \gamma(\mathbf{x}, \mathbf{w}) \mathrm{d} \mathbf{x} \\
=\lambda_{P E} L W \bar{\gamma}_{P E} \leq \lambda_{P E} L W \bar{\rho}_{P E}
\end{aligned}
$$

where (a) holds due to Campbell's theorem [18].

Then, by defining $\Theta=\Psi_{A E} \Psi_{A E}^{T}$, i.e., $\mathbf{w}=\Theta \mathbf{a}$, we can restate the optimization problem (9) as

$$
\begin{aligned}
& \arg \max _{\mathbf{a}} \varphi \mathbf{a}^{T} \mathbf{H}_{U}^{\Theta} \mathbf{a} \\
& \text { s.t. }\left\{\begin{array}{l}
\varphi \mathbf{a}^{T} \overline{\mathbf{H}}_{P E}^{\Theta} \mathbf{a} \leq \bar{\rho}_{P E} \\
|\Theta \mathbf{a}| \leq \mathbb{1}
\end{array}\right.
\end{aligned}
$$

where $\mathbf{H}_{U}^{\Theta}=\Theta^{T} \mathbf{H}_{U} \Theta$ and $\overline{\mathbf{H}}_{P E}^{\Theta}=\Theta^{T} \overline{\mathbf{H}}_{P E} \Theta$. Then, we form 
the Lagrangian as follows

$$
\begin{array}{r}
\mathcal{L}=\varphi \mathbf{a}^{T} \mathbf{H}_{U}^{\Theta} \mathbf{a}-\lambda\left(\varphi \mathbf{a}^{T} \overline{\mathbf{H}}_{P E}^{\Theta} \mathbf{a}-\bar{\rho}_{P E}\right) \\
+\mu_{-}^{T}(\Theta \mathbf{a}+\mathbb{1})-\mu_{+}^{T}(\Theta \mathbf{a}-\mathbb{1})
\end{array}
$$

where $\lambda \in \mathbb{R}$ and $\mu_{-}, \mu_{+} \in \mathbb{R}^{N}$ are the Lagrange multipliers. In order to allow $\mathcal{L}$ to have a non-trivial maximum value with respect to $\mathbf{a}$ and analytically calculate the solution of (11), the following condition must be satisfied:

$$
\mu_{-}=\mu_{+}=\mathbf{0} .
$$

Local sensitivity analysis [19] suggests that if this condition is satisfied, then the second constraint of (11b) is slack, i.e., $|\Theta \mathbf{a}|<\mathbb{1}$. The condition (13) can be satisfied depending on how high $\bar{\rho}_{P E}$ is set. If $\bar{\rho}_{P E}$ is set low, no element of $\Theta \mathbf{a}$ reaches \pm 1 , i.e., the second constraint is inactive. Here, it is worth mentioning that $\bar{\rho}_{P E}$ is a system parameter to be appropriately chosen according to given VLC environments, such as a particular distribution of LED transmitters, the location of the UE, the LED transmit power, etc. PLS theories recommend determining the value of $\bar{\rho}_{P E}$ such that it makes the SNR difference between the UE and the PEDs as large as possible given the VLC environments [11].

If condition (13) is satisfied, setting the partial derivative of $\mathcal{L}$ (with respect to a) equal to zero leads to

$$
\mathbf{H}_{U}^{\Theta} \mathbf{a}=\lambda \overline{\mathbf{H}}_{P E}^{\Theta} \mathbf{a} .
$$

Note that the rank of $\overline{\mathbf{H}}_{P E}^{\Theta}$ is less than or equal to $N-1$, i.e., it is singular. Hence, we utilize an inverse free preconditioned Krylov subspace projection method [12] to obtain the maximum eigenvalue $\lambda_{\max }$ and corresponding eigenvector $\mathbf{a}_{\max }$. In the method, an eigenvector estimate $\mathbf{a}_{k}$ ( $k$ is the iteration number) gradually grows through the Rayleigh-Ritz projection onto the Krylov subspace

$$
\begin{aligned}
& K_{m}\left(\mathbf{H}_{U}^{\Theta}-\rho_{k} \overline{\mathbf{H}}_{P E}^{\Theta}, \mathbf{a}_{k}\right) \\
& =\operatorname{span}\left(\mathbf{a}_{k},\left(\mathbf{H}_{U}^{\Theta}-\rho_{k} \overline{\mathbf{H}}_{P E}^{\Theta}\right) \mathbf{a}_{k}, \ldots,\left(\mathbf{H}_{U}^{\Theta}-\rho_{k} \overline{\mathbf{H}}_{P E}^{\Theta}\right)^{m} \mathbf{a}_{k}\right)
\end{aligned}
$$

where $\rho_{k}=\mathbf{a}_{k}^{T} \mathbf{H}_{U}^{\Theta} \mathbf{a}_{k} / \mathbf{a}_{k}^{T} \overline{\mathbf{H}}_{P E}^{\Theta} \mathbf{a}_{k}$ denotes the Rayleigh quotient, and $m$ is a parameter to be chosen. $\operatorname{span}\left(\mathbf{v}_{1}, \mathbf{v}_{2}, \ldots, \mathbf{v}_{N}\right)$ denotes the set of all linear combinations of a collection of vectors $\mathbf{v}_{1}, \mathbf{v}_{2}, \ldots, \mathbf{v}_{N}$. In this work, we utilize a MATLAB program eigifp implemented in [20] to conduct the entire procedure explained above, which takes the two inputs, $\mathbf{H}_{U}^{\Theta}$ and $\overline{\mathbf{H}}_{P E}^{\Theta}$ and returns the estimated maximum eigenvalue and corresponding eigenvector.

Then, the solution of (14) satisfies

$$
\gamma_{U}=\varphi \mathbf{a}^{T} \mathbf{H}_{U}^{\Theta} \mathbf{a}=\varphi \lambda \mathbf{a}^{T} \overline{\mathbf{H}}_{P E}^{\Theta} \mathbf{a} \leq \lambda_{\max } \bar{\rho}_{P E} .
$$

We can note that the SNR of the UE is proportional to the maximum eigenvalue $\lambda_{\max }$. Therefore, the optimal vector $\mathbf{a}^{*}$ can be obtained after scaling the eigenvector $\mathbf{a}_{\max }$ corresponding to $\lambda_{\max }$ such that $\varphi \mathbf{a}^{* T} \overline{\mathbf{H}}_{P E}^{\Theta} \mathbf{a}^{*}=\bar{\rho}_{P E}$. At last, the optimal ZF beamforming vector $\mathbf{w}^{*}$ can be obtained as $\mathbf{w}^{*}=\Theta \mathbf{a}^{*}$.

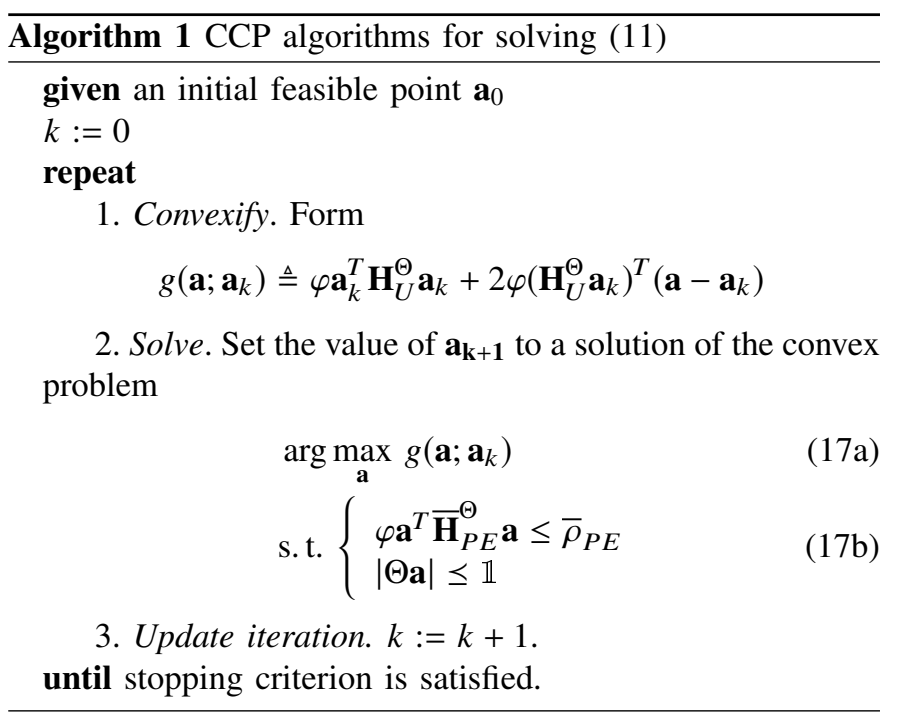

On the other hand, if any element of $\mathbf{w}^{*}$ obtained from the eigenmode of (14) is higher than 1 or less than -1 , the second constraint in (11b) is not slack; thus, (13) cannot be satisfied. In this case, the optimal vector $\mathbf{a}^{*}$ can be numerically found with the convex-concave procedure (CCP) [21], which is a powerful heuristic method to find local solutions. Given an initial feasible point $\mathbf{a}_{0}$, we transform the objective function (11a) into the affine function, i.e., convex, by applying the first-order Taylor series approximation. Then, we can solve the convex optimization problem by using a standard optimization programming like the sequential quadratic programming (SQP) algorithm [22], and set the solution to $\mathbf{a}_{1}$. The same procedure repeats until the improvement in the objective value is less than some threshold $\epsilon$. The detailed iterative algorithm is given in Algorithm 1.

\section{B. Minimizing Average SNR of Passive Eavesdroppers}

A related alternative objective may be to minimize the average SNR of the PEDs. This subsection investigates the optimization problem of finding a ZF beamforming vector with the objective, while satisfying the required SNR of the UE. Similar to the previous subsection, the optimization problem involving an arbitrary vector a can be formulated as

$$
\begin{aligned}
& \arg \min _{\mathbf{a}} \varphi \mathbf{a}^{T} \overline{\mathbf{H}}_{P E}^{\Theta} \mathbf{a} \\
& \text { s.t. }\left\{\begin{array}{l}
\varphi \mathbf{a}^{T} \mathbf{H}_{U}^{\Theta} \mathbf{a} \geq \rho_{U} \\
|\Theta \mathbf{a}| \leq \mathbb{1},
\end{array}\right.
\end{aligned}
$$

where $\rho_{U}$ denotes the required SNR of the UE.

By following the same approach in the previous subsection, an analogous result can be obtained as

$$
\mathbf{H}_{U}^{\Theta} \mathbf{a}=\eta \overline{\mathbf{H}}_{P E}^{\Theta} \mathbf{a}
$$

where $\eta=1 / \lambda$. Similarly, the maximum eigenvalue $\eta_{\max }$ and corresponding eigenvector $\mathbf{a}_{\max }$ can be obtained through the inverse free preconditioned Krylov subspace projection 
TABLE I. Simulation Parameters

\begin{tabular}{|c|c|}
\hline \multicolumn{2}{|l|}{ Room configuration } \\
\hline Length $(\mathrm{L}) \times$ Width $(\mathrm{W})$ & $10 \times 10 \mathrm{~m}^{2}$ \\
\hline Height from the work plane $(\mathrm{H})$ & $2.2 \mathrm{~m}$ \\
\hline Number of light fixtures & $5 \times 5$ \\
\hline Number of LEDs per fixture & 9 \\
\hline \multicolumn{2}{|c|}{ LED electrical and optical characteristics } \\
\hline Average optical power per LED & $0.72 \mathrm{~W}$ \\
\hline Optical power / current $\eta$ & 5 \\
\hline Nominal half-intensity angle $\Phi_{1 / 2}$ & $60^{\circ}$ \\
\hline Modulation index $\alpha$ & 0.5 \\
\hline \multicolumn{2}{|c|}{ Optical receiver characteristics } \\
\hline Photodetector's responsivity & $0.54 \mathrm{~mA} / \mathrm{mW}$ \\
\hline Lens refractive index $k$ & 1.5 \\
\hline Noise power $\sigma^{2}$ & $-98.33 \mathrm{dBm}$ \\
\hline Field of View $\Psi_{c}$ & $90^{\circ}$ \\
\hline
\end{tabular}

method, and the solution satisfies

$$
\bar{\gamma}_{E}=\varphi \mathbf{a}^{T} \overline{\mathbf{H}}_{P E}^{\Theta} \mathbf{a}=\varphi \frac{1}{\eta} \mathbf{a}^{T} \mathbf{H}_{U}^{\Theta} \mathbf{a} \geq \frac{1}{\eta_{\max }} \rho_{U} .
$$

From this, we can note that the minimum $\bar{\gamma}_{E}$ is inversely proportional to the maximum eigenvalue of $\eta_{\max }$. Thus, the optimal vector $\mathbf{a}^{*}$ can be obtained by scaling the corresponding eigenvector $\mathbf{a}_{\max }$ such that $\varphi \mathbf{a}^{* T} \mathbf{H}_{U}^{\Theta} \mathbf{a}^{*}=\rho_{U}$. Finally, the optimal beamforming vector $\mathbf{w}^{*}$ can be obtained as $\mathbf{w}^{*}=\Theta \mathbf{a}^{*}$.

On the other hand, if the second constraint in (18b) is active, the optimal solution $\mathbf{a}^{*}$ should be solved by a numerical method similarly to Algorithm 1, whereas the left term of the first constraint in (18b) needs to be transformed to an affine function (i.e., convex) by using the first-order Taylor series approximation as

$$
\varphi \mathbf{a}_{k}^{T} \mathbf{H}_{U}^{\Theta} \mathbf{a}_{k}+2 \varphi\left(\mathbf{H}_{U}^{\Theta} \mathbf{a}_{k}\right)^{T}\left(\mathbf{a}-\mathbf{a}_{k}\right) .
$$

\section{NumericAl Results}

In this section, numerical results are provided to verify the performance of the proposed ZF beamforming scheme. The room configuration and simulation parameters are provided in Table I. The $5 \times 5$ LED transmitters are uniformly distributed on a square lattice in a room of $10 \times 10 \mathrm{~m}^{2}$. The locations of the LED transmitters are given by $\left(x_{T}, y_{T}, 2.2\right)$, where $x_{T}$, $y_{T} \in\{0, \pm 2, \pm 4\}$.

Figs. 2(a) and (b) show the SNRs according to the receiver locations when the traditional ZF beamforming and the proposed ZF beamforming are adopted, respectively. The UE and the AED are located at $(1,1)$ and $(2.5,2.5)$, which are marked by blue and green dots, respectively. Also, $\bar{\rho}_{P E}=35 \mathrm{~dB}$ is used for the proposed ZF beamforming. For a fair comparison, the traditional ZF beamforming vector obtained from (6) is scaled such that it yields the average SNR of the PEDs as same as that of the proposed ZF beamforming. To aid presentation, the figures show the minimum SNR down to $-10 \mathrm{~dB}$, although the SNRs at the AED sites are much less than this value.

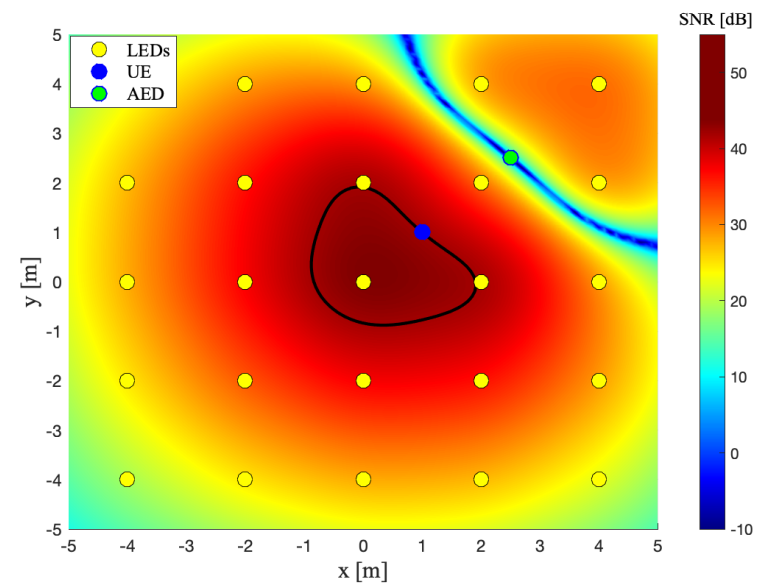

(a) The traditional ZF beamforming in [6]

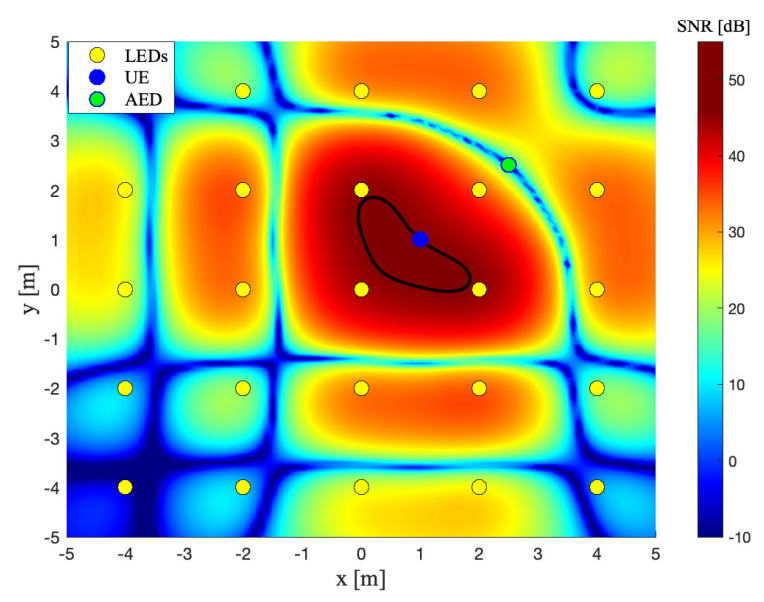

(b) The proposed ZF beamforming in (9)

Fig. 2. The SNR according to the receiver locations. The locations of the UE and the AED are $(1,1)$ and $(2.5,2.5)$, respectively. $\bar{\rho}_{E}=35 \mathrm{~dB}$ is used.

Besides, the black contours denote the area in which the SNR is higher than that of the UE. In the figures, it is noted that the SNR of the UE with the proposed ZF beamforming is $45.7 \mathrm{~dB}$, which is higher than that of the traditional ZF (41.9 dB). More importantly, thanks to the increased SNR of the UE, the proposed ZF beamforming can reduce the contour area to $7.29 \mathrm{~m}^{2}$, which is much smaller than the contour area with the traditional ZF beamforming $\left(20.74 \mathrm{~m}^{2}\right)$. The reduced contour area would result in a lower SOP. This result is because, unlike the traditional $\mathrm{ZF}$ beamforming that purely aims at suppressing the SNR of the AED to zero, the proposed ZF beamforming scheme is designed to maximize the SNR of the UE, simultaneously maintaining the SNR at the AED site as zero and constraining the average SNR of the PEDs.

Fig. 3 shows the SOPs obtained by Monte Carlo simulation for circles with different radii (centered at the UE) in which the AED is randomly present. This figure shows how the average distance between the UE and the AED affects the secrecy performance of the proposed ZF beamforming. The UE is 


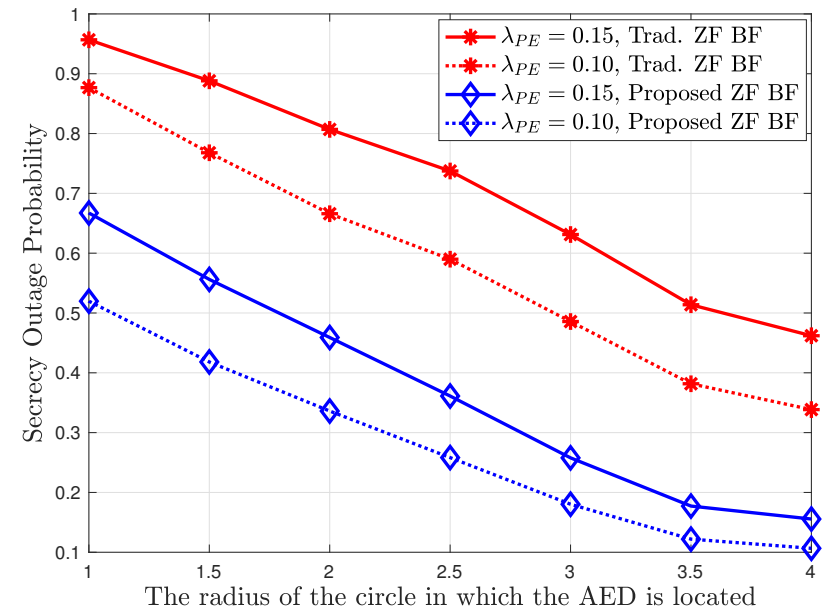

Fig. 3. The SOP plotted as a function of the radius of the circle in which the AED are randomly located. The locations of the UE is $(1,1)$, and the circle is centered at the UE. $\bar{\rho}_{P E}=35 \mathrm{~dB}$ is used.

located at $(1,1)$, and the AED is likely to be located closer to the UE when the circle radius is small. In the figure, it is shown that the proposed $\mathrm{ZF}$ beamforming scheme yields lower SOPs over the whole radii region than the traditional $\mathrm{ZF}$ beamforming. This result verifies that the proposed $\mathrm{ZF}$ beamforming scheme can effectively maximize the SNR of the UE, making it significantly larger than the traditional $\mathrm{ZF}$ beamforming method can as well as taking the active and passive EDs into account in the beamforming design. Furthermore, for both schemes, the SOP increases as the AED moves closer to the UE on average. For a nearby AED, the LED transmitters located close to the AED should appropriately use transmit power to beamform along the zero eigenmodes of the AED channel; thus, there is a limitation on maximizing the SNR of the UE. In contrast, when the AED is farther away from the UE, the LED transmitters located near to the UE can adequately adjust their beamforming weights to maximize the SNR of the UE without being hindered by the AED. Thus, a lower SOP can be achieved.

\section{CONCLUSION}

This paper proposed a ZF beamforming scheme to mitigate the effects of both an AED and hidden PEDs. Unlike the existing beamforming schemes that cope with either an AED or PEDs, but not both, the proposed ZF beamforming method can secure the VLC transmission against an AED and PEDs simultaneously. We formulated an optimization problem to find the ZF beamforming vector that lies in the null space of the AED's channel matrix so that the SNR of the AED can be nulled, while maximizing the SNR of the UE and constraining the average SNR of the PEDs. To find an eigenmode related to the optimal beamforming vector, we adopted the inverse free preconditioned Krylov subspace projection method. Reflecting on the fact that it may be the case that both PEDs and AEDs can be present in reality, it can be concluded that the proposed beamforming scheme provides a practical approach for engineering secure VLC systems.

\section{ACKNOWLEDGMENT}

This work was supported by EPSRC grant number EP/R006377/1 (“M3NETs").

\section{REFERENCES}

[1] P. Yang, Y. Xiao, M. Xiao, and S. Li, "6G wireless communications: Vision and potential techniques," IEEE Netw., vol. 33, no. 4, pp. 70-75, July 2019.

[2] Z. Zhang, Y. Xiao, Z. Ma, M. Xiao, Z. Ding, X. Lei, G. K. Karagiannidis, and P. Fan, "6G wireless networks: Vision, requirements, architecture, and key technologies," IEEE Trans. Veh. Technol., vol. 14, no. 3, pp. 28-41, Sep. 2019.

[3] L. U. Khan, "Visible light communication: Applications, architecture, standardization and research challenges," Digital Commun. Netw., vol. 3, no. 2, pp. $78-88,2017$.

[4] H. Haas, L. Yin, Y. Wang, and C. Chen, "What is LiFi?" J. Lightw. Technol., vol. 34, no. 6, pp. 1533-1544, Mar. 2016.

[5] A. Mostafa and L. Lampe, "Physical-layer security for MISO visible light communication channels," IEEE J. Sel. Areas Commun., vol. 33, no. 9, pp. 1806-1818, Sept. 2015.

[6] _ "Physical-layer security for indoor visible light communications," in IEEE ICC in Sydney, Australia, June 2014, pp. 3342-3347.

[7] T. V. Pham and A. T. Pham, "On the secrecy sum-rate of MU-VLC broadcast systems with confidential messages," in 2016 CSNDSP in Prague, Czech Republic, July 2016, pp. 1-6.

[8] T. V. Pham, H. Le-Minh, and A. T. Pham, "Multi-user visible light communication broadcast channels with zero-forcing precoding," IEEE Trans. Commun., vol. 65, no. 6, pp. 2509-2521, June 2017.

[9] S. Cho, G. Chen, and J. P. Coon, "Securing visible light communication systems by beamforming in the presence of randomly distributed eavesdroppers," IEEE Trans. Wireless Commun., vol. 17, no. 5, pp. 29182931, May 2018.

[10] _ "Enhancement of physical layer security with simultaneous beamforming and jamming for visible light communication systems," IEEE Trans. Inf. Forens. Security, vol. 14, no. 10, pp. 2633-2648, Oct. 2019.

[11] M. Bloch and J. Barros, Physical-Layer Security: From Information Theory to Security Engineering. Cambridge University Press, 2011.

[12] G. H. Golub and Q. Ye, "An inverse free preconditioned Krylov subspace method for symmetric generalized eigenvalue problems," SIAM J. Sci. Comput., vol. 24, no. 1, pp. 312-334, 2002.

[13] G. Geraci, S. Singh, J. G. Andrews, J. Yuan, and I. B. Collings, "Secrecy rates in broadcast channels with confidential messages and external eavesdroppers," IEEE Trans. Wireless Commun., vol. 13, no. 5, pp. 2931-2943, May 2014.

[14] T. Komine and M. Nakagawa, "Fundamental analysis for visible-light communication system using LED lights," IEEE Trans. Consum. Electron., vol. 50, no. 1, pp. 100-107, Feb. 2004.

[15] O. Ozel, E. Ekrem, and S. Ulukus, "Gaussian wiretap channel with an amplitude constraint," in IEEE Inf. Theory Workshop in Lausanne, Switzerland, Sept. 2012, pp. 5553-5563.

[16] S. Cho, G. Chen, H. Chun, J. P. Coon, and D. O'Brien, "Impact of multipath reflections on secrecy in VLC systems with randomly located eavesdroppers," in IEEE WCNC in Barcelona, Spain, Apr. 2018, pp. $1-6$.

[17] A. Baddeley, E. Rubak, and R. Turner, Spatial point patterns: methodology and applications with R. CRC Press, 2015.

[18] S. N. Chiu, D. Stoyan, W. S. Kendall, and J. Mecke, Stochastic geometry and its applications; 3rd ed., ser. Wiley Series in Probability and Statistics. Hoboken, NJ: Wiley, 2013.

[19] S. Boyd and L. Vandenberghe, Convex Optimization. New York, NY, USA: Cambridge University Press, 2004.

[20] J. Money and Q. Ye, "Algorithm 845: EIGIFP: a MATLAB program for solving large symmetric generalized eigenvalue problems," ACM Trans. Math. Softw., vol. 31, pp. 270-279, 012005.

[21] T. Lipp and S. Boyd, "Variations and extension of the convex-concave procedure," Optimization and Engineering, vol. 17, no. 2, pp. 263-287, June 2016.

[22] P. T. Boggs and J. W. Tolle, "Sequential quadratic programming," Acta Numerica, vol. 4, p. 151, 1995. 